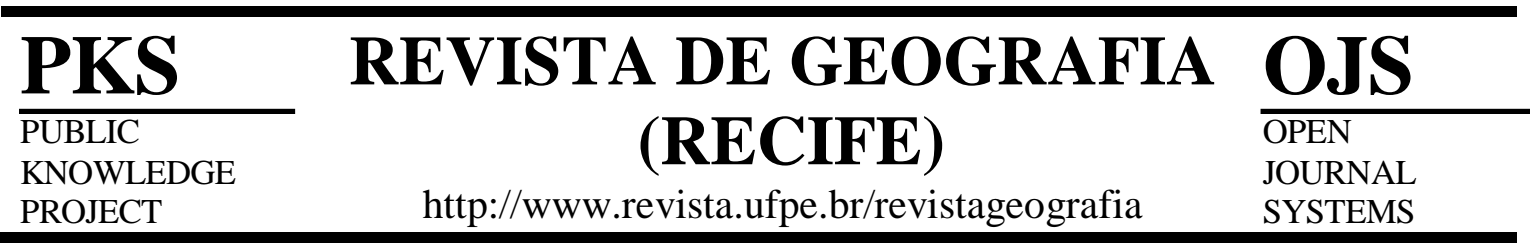

\section{CARACTERIZAÇÃO DE EXTREMOS ANUAIS DE PRECIPITAÇÃO PARA O ESTADO DE SERGIPE}

\author{
Washington Luiz Félix Correia Filho ${ }^{1}$; Laurizio Emanuel Ribeiro Alves ${ }^{2}$; Maurílio \\ Neemias dos Santos ${ }^{3}$
}

\author{
${ }^{1}$ Pós-doutorando em Ciências Climáticas pela PPGCC-UFRN. Email: wlfcfm@hotmail.com \\ ${ }^{2}$ Estudante de Graduação em Meteorologia pela UFAL e colaborador do SENSORGEO-UFAL. Email: \\ laurizio.r@gmail.com \\ ${ }^{3}$ Bacharel em Meteorologia (2015), atualmente faz parte do PPGMET-UFAL em Meteorologia, bolsista \\ do CNPq no laboratório SENSORGEO-UFAL.Email: maurilioneemias2010@gmail.com
}

Artigo recebido em 22/02/2016 e aceito em 09/09/2016

\begin{abstract}
RESUMO:
O agravamento das mudanças climáticas nos últimos anos tem propiciado numa maior ocorrência na quantidade dos eventos extremos, motivados por alterações no tempo e o clima global. Neste trabalho avaliaram-se os máximos anuais de precipitação e o índice de anomalia de chuva (IAC) por três estações pluviométricas do estado de Sergipe para o período de 1993 a 2015, a fim de caracterizar o padrão dos extremos via distribuição GEV (percentil 95) e a variabilidade anual entre anos secos / chuvosos. Verificou-se que os parâmetros de localização e de escala da distribuição GEV foram similares entre as estações utilizadas, entre 49,52mm e $60,95 \mathrm{~mm}$, e entre $19,70 \mathrm{~mm}$ e 22,83mm, respectivamente. Já o parâmetro de forma, as estações de Aracaju e Itabaianinha se ajustam a uma distribuição Fréchet, enquanto a estação de Propriá se ajusta a uma Weibull negativa. Os períodos de retorno previstos apontaram que chuvas acima de $150 \mathrm{~mm}$ (Aracaju) e entre 100-120 mm (Itabaianinha e Propriá) para os próximos 5-10 anos. Os valores de IAC foram negativos em ano subsequente a ocorrência de El Niño, no entanto, no La Niña de intensidade moderada ocorrido em 2011, no ano de 2012 registrou o maior IAC negativo do período de estudo.
\end{abstract}

Palavras-chave: Índice de Anomalia de Chuva, Distribuição GEV, Aracaju

\section{ASSESSMENT OF ANNUAL VARIABILITY AND PRECIPITATION EXTREMES FOR SERGIPE STATE}

\begin{abstract}
:
The worsening of climate change in recent years has resulted in the higher incidence of extreme events, driven by changes in the weather and the global climate. In this work, it was evaluated the maximum annual precipitation and the Rainfall Anomaly Index (RAI) for three rainfall stations of the Sergipe state for the period 1993-2015 was calculated in order to characterize the pattern of extremes by GEV distribution $\left(95^{\text {th }}\right.$ percentile) and the annual variability between dry / wet years. The location and scale parameters of the GEV distribution were similar between stations, between $49.52 \mathrm{~mm}$ and $60.95 \mathrm{~mm}$ and between $19.70 \mathrm{~mm}$ and $22.83 \mathrm{~mm}$, respectively. The shape parameter of stations of Aracaju and Itabaianinha fits a Fréchet distribution, whereas Propriá station fits a negative Weibull distribution. Periods of planned return indicate that rainfall will be above $150 \mathrm{~mm}$ (Aracaju) and between $100-120 \mathrm{~mm}$ (Itabaianinha and Propriá) for the next 5-10 years. The IAC values were negative in the year following the El Niño occurrence, however, the La Niña of moderate intensity occurred in 2011, on year 2012 recorded the highest negative IAC on study period.
\end{abstract}

Keywords: Rainfall Anomaly Index; Generalized Extreme Value Distribution; Aracaju. 


\section{INTRODUÇÃO}

A tentativa de melhor compreender os efeitos das mudanças climáticas nos ciclos de precipitação em regiões com regimes irregulares é importante, visto a necessidade da comunidade local pela demanda hídrica sob as mais diversas atividades socioeconômicas e ambientais (BRAGA et al., 2006).

$\mathrm{Na}$ agricultura, o conhecimento do período chuvoso é essencial ao produtor, que com base nas informações meteorológicas realize um bom planejamento agrícola, evitando perdas provenientes da necessidade da cultura em período de plantio (PELLEGRINO et al., 2006).

Outro exemplo da necessidade do monitoramento das chuvas, está associado ao abastecimento de energia e água de uma cidade, tendo em vista a forte dependência do Brasil na geração de usinas hidroelétricas. É fundamental a detecção antecipada dos possíveis fatores que alterem no regime das chuvas, e posteriormente dos rios ocasionadas por fatores climáticos, e a partir de políticas públicas busquem minimizar os impactos destes efeitos sobre a população (TUCCI, 2007; MARENGO, 2008).

Quando se avalia as chuvas na região Nordeste do Brasil (NEB), a principal característica relacionada ao tema é a forte irregularidade das chuvas entre o litoral e o semiárido (DINIZ, 2014), com precipitação média anual ao longo do NEB varia entre $1800 \mathrm{~mm}$ na região costeira, em torno de $1400 \mathrm{~mm}$, enquanto que no centro do semiárido apresenta em média apenas 400mm (SILVA et al., 2012).

De acordo com Diniz (2014), o clima do nordeste brasileiro apresenta uma grande variabilidade interanual das chuvas, pois apresenta anos de seca extrema e extremamente chuvosos. A variabilidade das chuvas pode ocorrer de duas maneiras: interanual ou interanual.

Em ambas as situações, alguns fatores são determinantes, por exemplo: período de ocorrência, intensidade, tempo de vida e a região de influência dos sistemas meteorológicos, que por sua vez, atuam de forma distinta sobre a região Nordeste do Brasil (NEB). Dentre os principais sistemas podemos citar: Vórtice Ciclônico de Ar Superior (VCAS), Zona de Convergência Intertropical (ZCIT), Distúrbios Ondulatórios de Leste (DOL) (MOLION \& BERNARDO, 2002; LIEBMANN et al., 2011; CAVALCANTI, 2012).

Estes sistemas meteorológicos são fortemente influenciados pelas regiões tropicais das bacias oceânicas do Atlântico e Pacífico (ANDREOLI \& KAYANO, 
2006; KUCHARSKI et al., 2008; KAYANO et al., 2013), associados principalmente à circulação de grande escala (MOLION \& BERNARDO, 2002).

Sob o aspecto da variabilidade interanual podemos citar o El Niño-Oscilação Sul (ENOS), um dos grandes responsáveis pela modulação do tempo e clima global, possui um ciclo de ocorrência entre 3 a 7 anos, determinante no padrão de chuvas no NEB (EASTERLING et al., 2000; MEEHL \& TEBALDI, 2004; TRENBERTH, 2011; TRENBERTH, 2012).

Estas alterações verificadas a partir dos principais sistemas moduladores do clima global motivadas pela intensificação das mudanças climáticas têm contribuído para a recorrência de extremos climáticos mais frequentes e intensos (TOMINAGA, 2009), tornando-se uma das grandes preocupações da comunidade internacional quando se refere ao contexto climático.

Estes impactos ocasionados por eventos como secas severas e inundações se tornaram o mais frequentes, assim regiões com maior vulnerabilidade sofrerão os maiores impactos (EASTERLING et al., 2000; MEEHL \& TEBALDI, 2004; TRENBERTH, 2011; TRENBERTH, 2012).

A implementação de novas metodologias tem contribuído na caracterização dos extremos, um destes métodos são os índices climáticos, baseada em índices de extremos climáticos criados pela Organização Meteorológica Mundial (WMO, 2009; SKANSI et al., 2013), com a finalidade de verificar a tendência e sazonalidade dos eventos.

Outro método utilizado é a Teoria dos Valores Extremos (TVE) (FENG et al., 2007; IDALINO et al., 2009; SCARROT \& MACDONALD, 2012), baseadas na aplicação das distribuições generalizadas de valores extremos (GEV) e de Pareto (GPD), extraindo características dos extremos (média, desvio e o tipo de distribuição que a série se ajusta).

A TVE é comumente aplicada em dados de temperatura e de precipitação, devido à sensibilidade de ambas para qualquer alteração sutil no clima pode ser facilmente perceptível por ambas as variáveis (SCARROT \& MACDONALD, 2012).

Outra forma utilizada para a detecção dos extremos de precipitação é o Índice de Anomalia de Chuva (IAC), a partir de valores mensais ou anuais é possível identificar os extremos climáticos por excesso / escassez. Este índice foi desenvolvido por Rooy (1965), sendo este simples e fácil aplicabilidade, pois a extração dos valores de anomalias extremas depende apenas da precipitação. 
O IAC pode ser calculado em diversas escalas de tempo, sendo esta semanal, mensal ou anual. Após a identificação dos extremos climáticos, ocorrência de período extremamente seco e chuvoso. Sua eficácia em caracterizar regiões climáticas, com o propósito de detectar os extremos de precipitação é constatada em diversas pesquisas, inclusive no NEB (REPELLI et al. 1998; KEYANTASH, 2002; FREITAS, 2005; DOS SANTOS, 2013; SANTOS et al., 2015).

Com base nas informações apresentadas, o objetivo deste trabalho será avaliar a precipitação no Estado de Sergipe entre os anos de 1993-2015 por duas abordagens: $1^{\text {a }}$ ) em escala diária, diagnosticar o comportamento dos máximos diários durante o período chuvoso (fevereiro a julho) via distribuição GEV (percentil 95); e $2^{\mathrm{a}}$ ) em escala anual, diagnosticar os anos chuvosos / secos a partir do IAC, com base nas anomalias anuais.

\section{MATERIAL E MÉTODOS}

\section{1.Área de estudo}

O estado de Sergipe está situado no Nordeste brasileiro e têm como limites o Oceano Atlântico a leste, o estado da Bahia a oeste e sul e o estado de Alagoas ao norte, do qual está separado pelo Rio São Francisco (latitude $10^{\circ} 54^{\prime} 40^{\prime}$ ' Sul, longitude $37^{\circ}$ 04' 18" Oeste) (Figura 1). Considerado o menor estado brasileiro, Sergipe possui dimensões territoriais que ocupa uma área de $21.918,493 \mathrm{Km}^{2}$, com uma população estimada de 2.068,017 habitantes (IBGE, 2010).

As principais atividades econômicas do estado são: agricultura, pecuária e extrativismo mineral (petróleo e gás natural). O relevo do estado de Sergipe apresenta predominância de áreas planas e / ou onduladas, com altitude inferior a 300 metros, além disso, cerca de aproximadamente $50 \%$ do território está inserido no polígono das secas (GURJEL, 1986).

De acordo com Pereira et al. (2011), o regime pluviométrico do Estado de Sergipe está sob influências de sistemas atmosféricos distintos que atuam nos setores Norte e Leste do Nordeste do Brasil (NEB), ao qual possui uma característica de transição entre os regimes pluviométricos da parte Norte (com máximos de fevereiro a maio) e Sul (dezembro a fevereiro) do NEB. 


\subsection{Dados Utilizados}

Neste estudo foram utilizados dados diários de três estações meteorológicas do período de 01 de Janeiro de 1993 à 31 de Dezembro de 2015, dos quais três são convencionais pertencentes ao Instituto Nacional de Meteorologia (INMET) e vinculadas à Organização Mundial de Meteorologia (OMM): Aracaju (OMM: 83096, Latitude - 10,95 ${ }^{\circ} \mathrm{S}$; Longitude - 37,04 ${ }^{\circ} \mathrm{W}$; Altitude - 4,72m); Itabaianinha (OMM 83195, Latitude - $11,11^{\circ} \mathrm{S}$; Longitude - $37,81^{\circ} \mathrm{W}$; Altitude - 208m); e Própria (OMM 83097, Latitude - $10,21^{\circ} \mathrm{S}$; Longitude - 36,84 ${ }^{\circ} \mathrm{W}$; Altitude - 19,92m), disponíveis no Banco de Dados Meteorológicos para Ensino e Pesquisa (BDMEP) no sítio http://www.inmet.gov.br/portal/index.php?r=bdmep/bdmep. As estações utilizadas neste estudo estão identificadas na Figura 1.

As três estações meteorológicas estão distribuídas em locais distintos, a estação de Aracaju se encontra próximo ao litoral no leste sergipano, Itabaianinha está localizada na divisa entre agreste e sertão de Sergipe, e Propriá se encontra no leste sergipano, às margens do Rio São Francisco na divisa entre Sergipe e Alagoas.

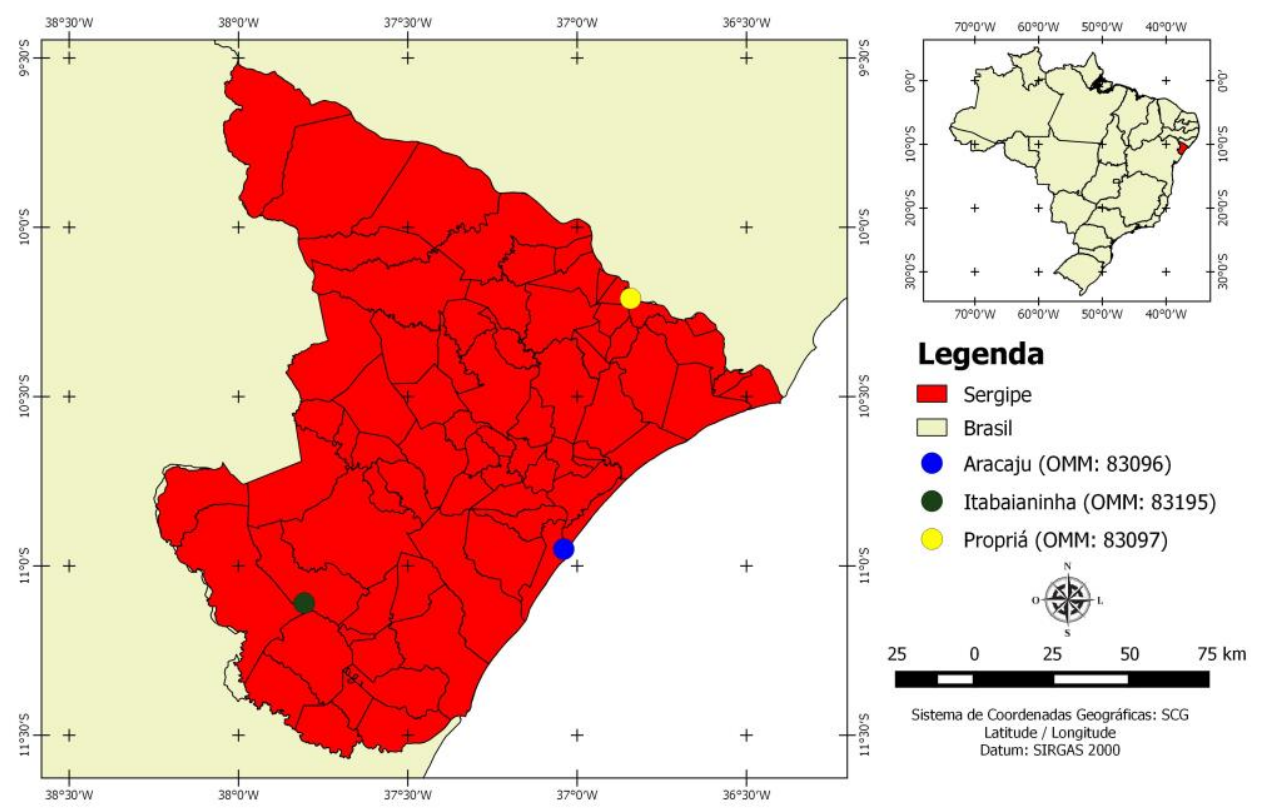

Figura 1 - Localização das três estações meteorológicas do INMET. 


\subsection{Teoria dos Valores Extremos (TVE)}

A modelagem da Teoria dos Valores Extremos (TVE) via distribuição Generalizada de Valores Extremos (GEV) inicia-se a partir de uma série de observações independentes $\left(X_{1}, \ldots, X_{n}\right)$ limitados a uma sequência ou intervalo de observações de comprimento $n$, para cada valor máximo de $m$ gera-se uma série máxima por bloco ou período $\left(X_{p, 1}, \ldots, X_{m, n}\right)$, ao qual estão contidas em detalhes em Coles et al. (2001) e Beirlant et al. (2006).

Uma vez determinado o comprimento destes blocos ou do período, por exemplo, o bloco máximo anual, $n$ será o número de observações no ano para a obtenção dos máximos anuais por blocos. A escolha do tamanho do bloco ou período é essencial para a estimação dos parâmetros da distribuição GEV, e também para o nível de retorno, pois se for muito pequeno interferirá na estimação do viés e extrapolação, e se muito grande gerariam poucos blocos de máximos, resultando no aumento da variância. Este processo permite uma renormalização linear da variável $M_{n}$ :

$M_{n}^{*}=\frac{M_{n}-b_{n}}{a_{n}}$,

Com o aumento de $n$, quando $a_{n}>0$ e $b_{n}$ permanecem constantes, tem-se a seguinte expressão para $\operatorname{Pr}\left\{M_{n}-b_{n} / a_{n} \leq z\right\} \rightarrow G(z)$ como $n \rightarrow \infty$. Sendo G, uma função de distribuição não-degenerada pertencente a uma das famílias que seguem abaixo:

$I: G(z)=\exp \left\{-\exp \left[-\left(\frac{z-\mu}{\xi}\right)\right]\right\},-\infty<z<\infty$

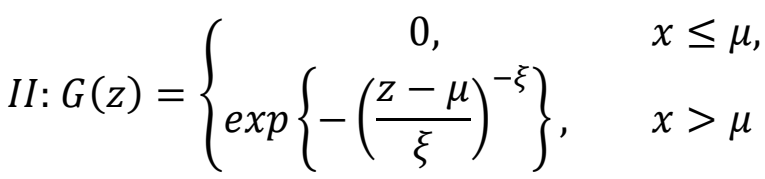

III: $G(z)=\left\{\begin{array}{cl}\exp \left\{-\left(\frac{z-\mu}{\xi}\right)^{-\xi}\right\}, & x<\mu, \\ 1, & x \geq \mu,\end{array}\right.$ 
De forma geral, estas distribuições de classe são chamadas de distribuição de valores extremos dos tipos I, II e III conhecidas como famílias: Gumbel ( $\square \square \square$ ), Fréchet $(\square \square \square)$ e Weibull negativa $(\square \square \square)$, respectivamente.

Os parâmetros da distribuição GEV demonstram as principais características sobre o padrão da série de extremos: localização $(\square)$ indica o valor médio do extremo; escala ( $\square$ ) informa a amplitude da dispersão da série; e forma ( $\square$ ) define qual distribuição a série pode ser ajustada. Estas famílias possuem parâmetros de localização $(\square)$, escala ( $\square$ ) e forma $(\square)$ distintas, com aplicações diferenciadas para cada família, exibindo diferentes comportamentos de valor extremo (CORREIA FILHO, 2014).

A forma simplificada da distribuição proposta por Jenkinson (1955) pode ser descrita pela expressão abaixo:

$G(z)=\exp \left\{-\left[1+\xi\left(\frac{z-\mu}{\sigma}\right)\right]^{-\frac{1}{\xi}}\right\}$

Pelo ajuste $\{z: 1+\xi(z-\mu / \sigma)>0\}$, os parâmetros satisfazem $-\infty<\mu<0$ e $-\infty<\xi<\infty$, com os parâmetros de localização $(\mu)$, de escala $(\sigma)$ e de forma $(\xi)$.

A estimação do período e nível de retorno dos extremos a partir da distribuição GEV é obtida pela inversão da Equação 2:

$$
Z_{p}=\left\{\begin{array}{c}
\mu-\frac{\sigma}{\xi}\left\{1-[\log (1-p)]^{-\xi}\right\} \\
\mu-\sigma \log [-\log (1-p)]
\end{array}\right.
$$

Em que $G\left(z_{p}\right)=1-p \cdot z_{p}$ é o nível de retorno em relação ao período de retorno $1 / p$, $p$ é a probabilidade de ocorrência do extremo, caso os máximos por blocos seja anual, $z_{p}$ será dado em anos.

De acordo com Folland et al. (1999) é preferível a utilização dos percentis a valores dos limiares absolutos, devido a heterogeneidade das áreas e / ou processos físicos que mascarem o resultado. A qualidade de ajuste do modelo gerada pela GEV pode ser avaliada pelo diagnóstico de diversos gráficos: probabilidade, quantis, densidade e de níveis de retorno. 


\section{4. Índice de Anomalia de Chuva (IAC)}

Para o diagnóstico do comportamento de períodos secos e chuvosos ao longo dos vinte e dois anos, utilizamos o IAC, um método simplificado e objetivo que avalia a variabilidade espaço-temporal de precipitação, apenas utiliza dados de precipitação, utilizado em diversos estudos no NEB (REPELLI et al. 1998; KEYANTASH, 2002; FREITAS, 2005; DOS SANTOS, 2013; SANTOS et al., 2015).

A classificação do IAC é realizada com base em vinte eventos anuais: dez (10) secos e dez (10) eventos chuvosos, respectivamente. O IAC analisa a frequência da ocorrência de anos secos e chuvosos, além disso, seu grau de severidade e a duração são obtidas pelas Equações 3 e 4:

$I A C=3\left[\frac{P_{t}-P_{m}}{X_{l}-P_{m}}\right]$, para anomalias positivas (Períodos Chuvosos)

$I A C=-3\left[\frac{P_{t}-P_{m}}{X_{m}-P_{m}}\right]$, para anomalias negativas (Períodos secos)

Em que o $\mathrm{P}_{\mathrm{t}}$ compreende a precipitação total do ano $(\mathrm{mm})$ que será calculado o $I A C, P_{m}$ corresponde a precipitação média anual da série histórica $(\mathrm{mm}), X_{l}$ e $X_{m}$ correspondem à média dos dez maiores e menores valores de precipitação anual da série histórica $(\mathrm{mm})$, respectivamente.

As anomalias positivas (negativas) do IAC são representadas por valores acima (abaixo) da média histórica, de acordo com o grau de intensidade das anomalias descrito na Tabela 1.

Tabela 1 - Faixa de Classificação do Índice de Anomalia de Chuva (IAC).

Fonte: Fernandes (2009).

\begin{tabular}{cc}
\hline Valores do IAC & Classificação \\
\hline$\geq 4,00$ & Extremamente úmido \\
3,00 a 3,99 & Umidade alta \\
2,00 a 2,99 & Umidade Moderada \\
0,50 a 1,99 & Umidade Baixa \\
$-0,49$ a 0,49 & Normal \\
$-1,99$ a $-0,50$ & Seca suave \\
$-2,00$ a $-2,99$ & Seca moderada \\
$-3,00$ a $-3,99$ & Seca alta \\
$\leq-4,00$ & Seca extrema \\
\hline
\end{tabular}




\section{RESULTADOS E DISCUSSÃO}

\subsection{Análise descritiva dos dados anuais de precipitação}

Os resultados apresentados a seguir avaliam o comportamento da distribuição de frequência dos dados diários das três estações oriundas do Estado de Sergipe (Aracaju, Itabaianinha e Propriá) exibido na Figura 2, e representado nas Figuras 3 a 5, por boxplots anuais para cada estação, correspondentes ao período chuvoso (março a junho) do período de 1993 a 2015.
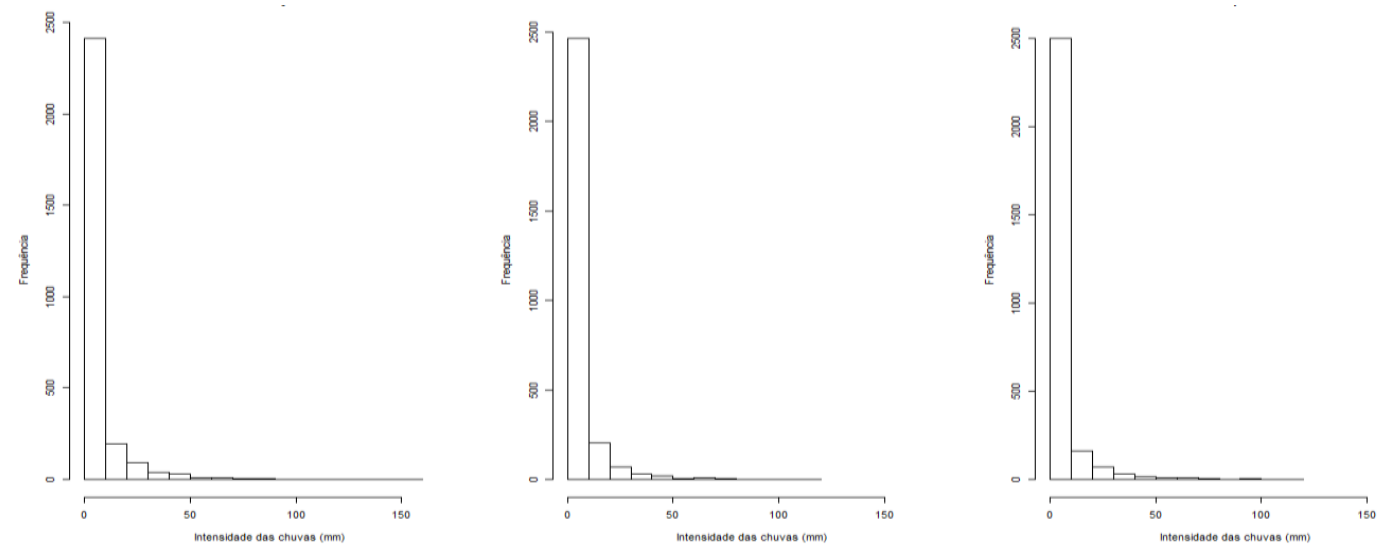

Figura 2 - Histograma do período chuvoso (março a junho) para o período de 1993-2015 das estações de Aracaju (à esquerda), Itabaianinha (ao centro) e Propriá (à direita).

De maneira geral, mesmo diante da particularidade de as três estações exibirem características climáticas bem distintas umas das outras, no entanto, as mesmas apresentam histogramas com características similares, as distribuições de frequência dos dados concentram-se em quase sua totalidade na faixa de valores inferiores à $10 \mathrm{~mm}$, contabilizando um número que varia entre 2400 a 2500 dias, reduzindo consideravelmente a frequência do número de dias com chuvas de intensidades superiores à $10 \mathrm{~mm}$.

Observando os boxplots das precipitações diárias anuais para cada uma das três estações utilizadas neste trabalho, é possível identificar que em alguns anos os valores médios da quadra chuvosa são muito baixos, com valores máximos inferiores à $20 \mathrm{~mm}$. No entanto, também se verifica que em todos os anos, uma grande quantidade de dados fora do limite superior do boxplot, ou seja, outliers. 
No caso da estação de Aracaju (Figura 3), verifica-se a presença de outliers de maior intensidade entre os anos de 1993 a 1999, com valores máximos diários de chuva superiores a $60 \mathrm{~mm} / \mathrm{dia}$, no entanto, entre os anos de 2000-2010, nota-se uma diminuição quanto aos valores outliers de chuva, atingindo $60 \mathrm{~mm} / \mathrm{dia}$, com exceção do ano de 2008 que registrou um extremo de $110 \mathrm{~mm} /$ dia.

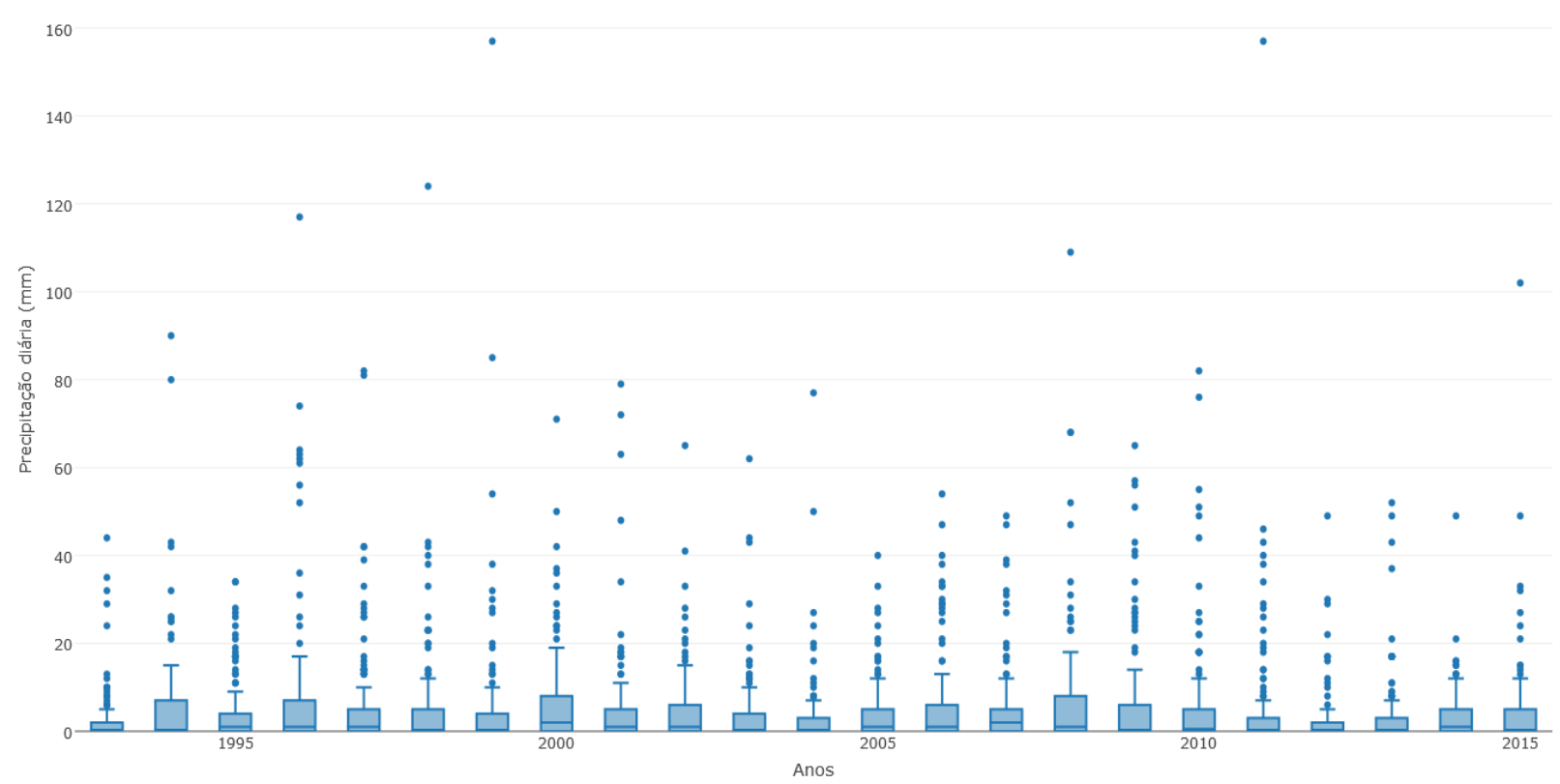

Figura 3 - Boxplot da precipitação diária anual do município de Aracaju para o período chuvoso entre os anos de 1993-2015.

Verifica-se também que em alguns anos, os valores máximos dos outliers não ultrapassam os 60 mm/dia, são os casos dos anos de 1993, 1995, 1997, de 2005 a 2007, de 2011 a 2014. Com relação a estação de Itabaianinha, constata-se algumas diferenças em relação à Aracaju, inicialmente o número de outliers acima de $60 \mathrm{~mm} /$ dia é um pouco menor, mas tem-se registrado extremos diários de chuva acima dos $80 \mathrm{~mm} /$ dia entre os anos de 2012 e 2015. Verifica-se também que em alguns períodos os valores máximos dos outliers não ultrapassam os 60 mm/dia em alguns anos, como os períodos de 1993 a 1995, 1999 a 2002, 2004 e 2005 , e 2010 a 2012. 


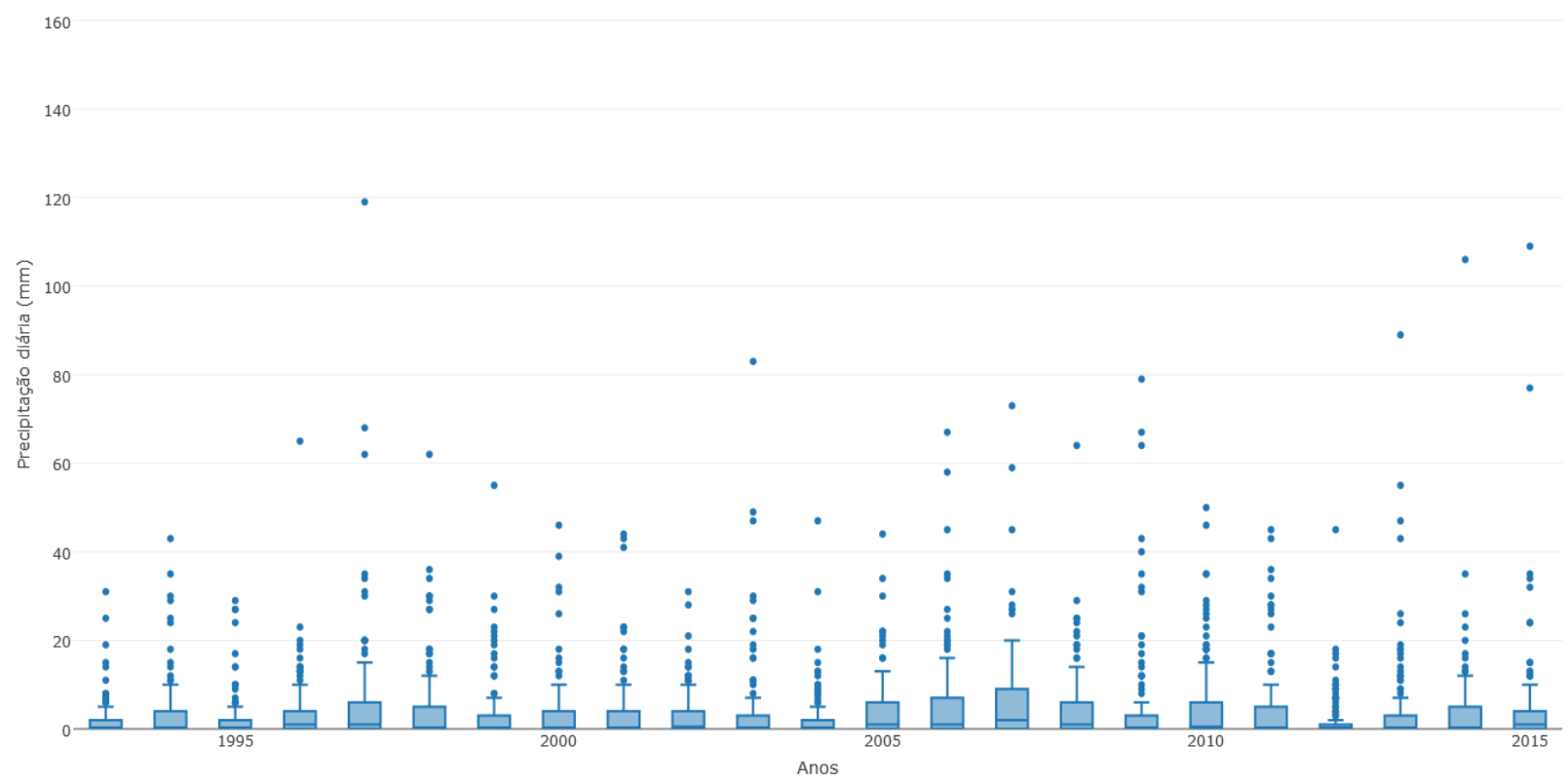

Figura 4 - Boxplot da precipitação diária anual do município de Itabaianinha para o período chuvoso entre os anos de 1993-2015.

Avaliando a estação de Propriá, nota-se um comportamento distinto em relação as duas estações descritas anteriormente, a primeira distinção está relacionada a grande maioria dos máximos anuais de seus outliers serem inferiores a $60 \mathrm{~mm} / \mathrm{dia}$, como os períodos de 1993, 1995, 1998 a 2000, 2002 a 2005, 2008, e 2012 a 2014.

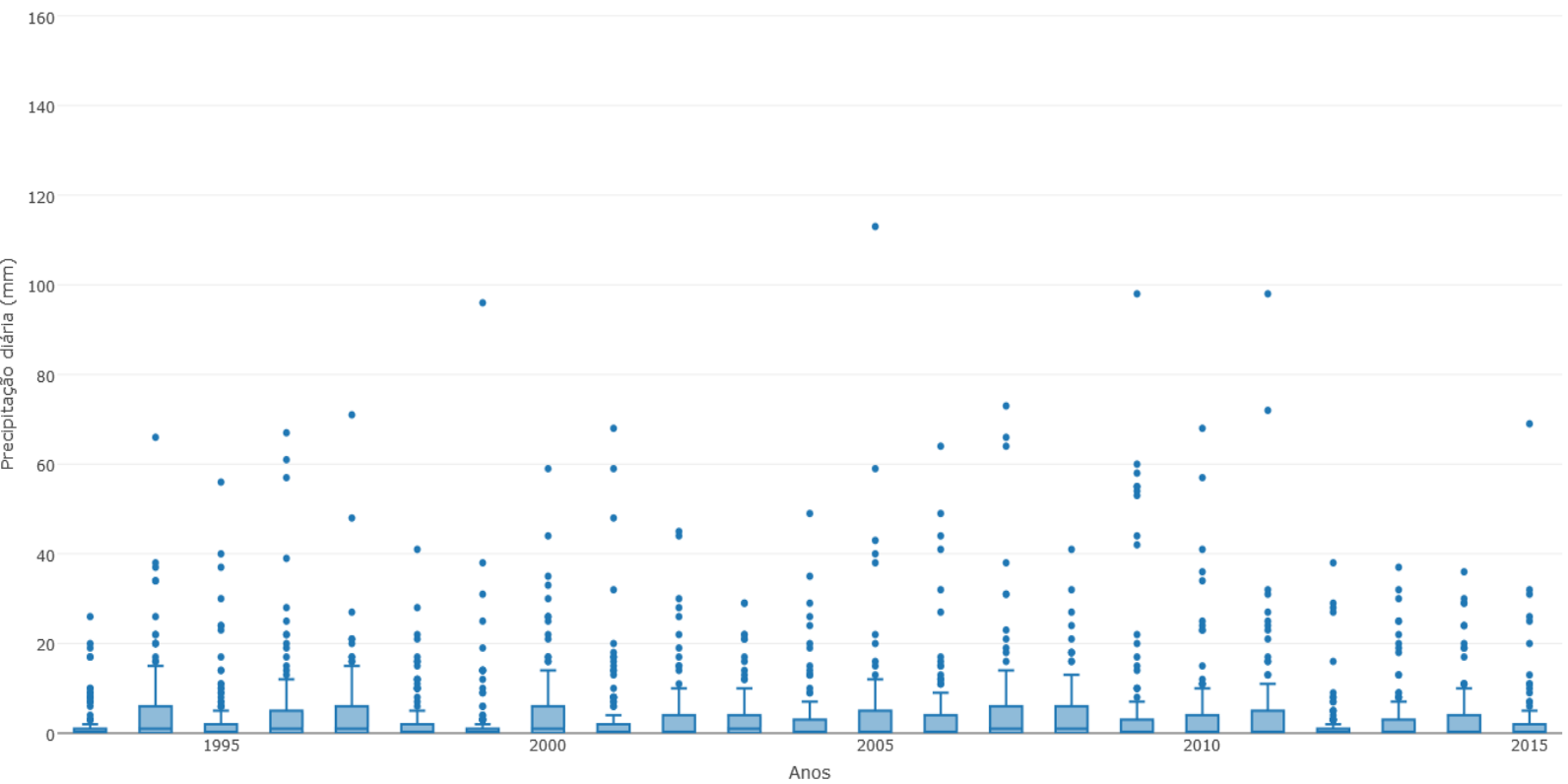


Figura 5 - Boxplot da precipitação diária anual do município de Propriá para o período chuvoso entre os anos de 1993-2015.

Avaliando a variabilidade dos acumulados de precipitação anual no estado de Sergipe (Figura 6) durante o período chuvoso, entre o período de 1993 a 2015, constata-se a existência de ciclos de valores de mínima, em torno de aproximadamente entre 3-5 anos, durante os anos de 1993, 1995, 1998, 2002, 2008, 2012 e 2015, com maior evidência no município de Propriá, ao qual os valores variam entre $600 \mathrm{~mm}$ (ano de 2012) e $1300 \mathrm{~mm}$ (ano de 2000).

Estas oscilações existentes nos totais anuais de precipitação para o período chuvoso, também foram constatadas nos boxplots (Figuras 3,4 e 5), ao qual a amplitude entre os valores extremos de mínimas e máximas eram pequenos, indicando uma menor variabilidade. Já a estação de Itabaianinha apresenta aumento sistemático no índice pluviométrico entre os anos de 1993 a 2000, entre $600 \mathrm{~mm}$ e $1200 \mathrm{~mm}$, no entanto, dentro da série anual os menores valores anuais de precipitação ocorrem nos anos de 1993, 2012 e 2015.

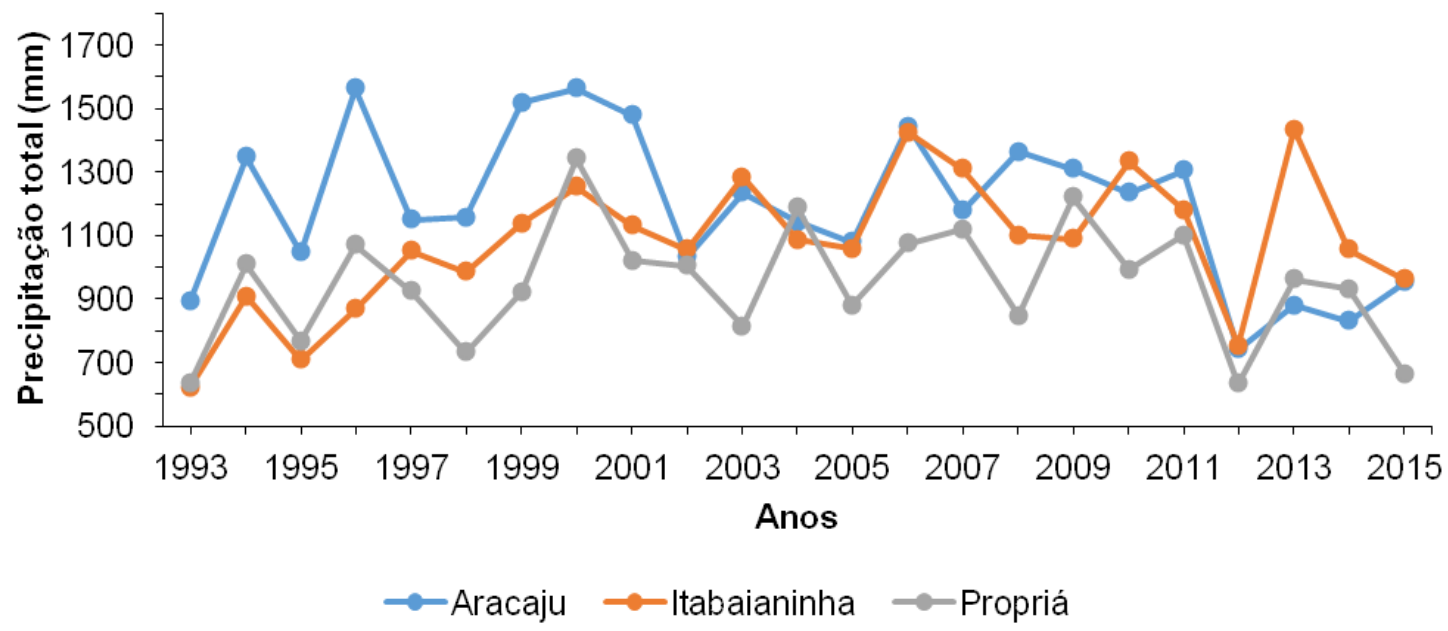

Figura 6 - Série temporal anual da precipitação entre os anos de 1993-2015, para as estações de Aracaju, Itabaianinha e Propriá.

Estes resultados dos registros mínimos totais anuais de precipitação apontam a influência ocorrência de El Niño-Oscilação Sul. Verifica-se que em anos posteriores a ocorrência de El Niños, há uma diminuição no total de chuvas e também em seus extremos, exibidos nos anos de 1993, 1995, 1998, 2003, 2008 e 2015, o que corrobora com os resultados 
apresentados por De Souza Gurjão et al. (2012), que nos anos de 1998 e 2008 ocorreram reduções significativas nos índices pluviométricos.

No entanto, devido ao La Niña moderado ocorrido em 2011, no ano de 2012 nota-se uma redução significativa nos índices pluviométricos nas três estações de estudo, tendo em vista que este comportamento do La Niña é incomum, por estar associado a melhor distribuição das chuvas no NEB. Este resultado corrobora com o mesmo o resultado apresentado por Rodrigues \& McPhaden (2014), este comportamento anômalo é decorrente de anomalias de positivas de temperatura da Superfície do Mar sobre o Oceano Atlântico Tropical Sul, resultando neste efeito contrário.

\section{2. Índice de Anomalias de Chuva (IAC)}

Os resultados apresentados a seguir são referentes ao IAC anual para as três estações meteorológicas, apresentadas na Figura 7. Nota-se uma forte similaridade entre as estações em alguns anos (1993, 1995 e 2012), exibindo características de secas moderadas à extremas (valores inferiores ao IAC $=-2$ ), sendo mais severo na cidade de Itabaianinha que demonstra em alguns anos seca extrema, este resultado está em consonância com apresentado por De Souza Gurjão et al. (2012), ao qual também captaram valores de IAC próximos a -2 no ano de 1998, em Sergipe.

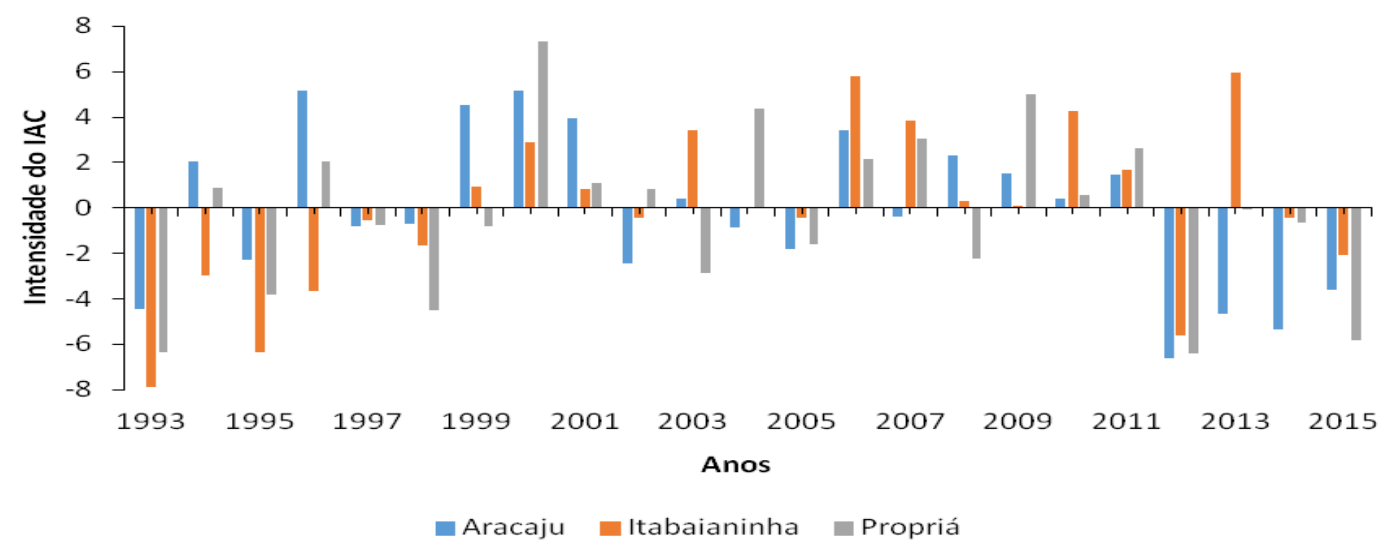

Figura 7 - Variabilidade anual do AIC entre os anos de 1993-2015, para as estações de Aracaju, Itabaianinha e Propriá. 
Quanto à variabilidade do IAC ao longo dos vinte e dois anos para as três estações, verifica-se que em Aracaju a seca extrema ocorre no ano de 2012, com valor inferior de -6,64, e extremidade úmida no ano de 1996, com IAC de 5,16. Já Itabaianinha observa seca extrema no ano de 1993, com IAC de -7,87, constatando o maior valor negativo de IAC entre as três séries analisadas, enquanto o IAC positivo ocorre em 2013, com o calor de 5,94, caracterizado como extremamente úmido.

Este período de seca constatado em 2012, é resultante do La Niña moderado, constatado por Rodrigues \& McPhaden (2014), já em 1993 foi decorrente do forte El Niño de 1992, ao qual resultaram em valores extremos negativos de IAC, corroborando com os resultados de Rao et al. (1995), que evidenciaram a ocorrência de seca severa, motivada pelo forte El-Niño de 1991-1992.

Em Propriá, localizado à beira do Rio São Francisco, exibe seca extrema no ano de 2012, comportamento semelhante à estação de Aracaju, como valor negativo de IAC de 6,42, enquanto é extremamente úmido foi observado no ano 2000, com o valor positivo de IAC de 7,35, sendo o maior índice positivo entre as três estações analisadas. Um destaque interessante é que durante a ocorrência do forte El Niño de 1997-1998 e moderado de 2002-2003, as três estações do estudo exibiram secas suaves, muito diferentes do padrão apresentado ao longo do NEB que sofrem com secas intensas, verificados nos resultados apresentados por Kucharski et al. (2008).

\subsection{Ocorrência de extremos via GEV}

A seguir serão discutidos os resultados do diagnóstico de extremos diários anuais de precipitação obtidos pela distribuição GEV (percentil 95) para o período chuvoso (março a junho) para as estações meteorológicas de Aracaju, Itabaianinha e Propriá. Verifica-se que os valores referentes aos parâmetros de localização ( $\square$ ), escala ( $\square$ ) e forma ( $\square$ ) são similares, mesmo diante da não proximidade das estações e também a influência topográfica da estação de Itabaianinha com seus 208 metros de altitude, as três apresentam homogeneidade (contidas na Tabela 2).

Os valores do parâmetro de localização $(\square)$ variam entre 49,52mm (Itabaianinha) e $60,95 \mathrm{~mm}$ (Aracaju). O parâmetro de escala $(\square)$ variou em torno de 19,70mm (Itabaianinha) e 
22,83 mm (Aracaju). No entanto, quando nos referimos a intensidade máxima diária anual, a estação de Aracaju exibe o maior valor com $157 \mathrm{~mm}$. Outro detalhe a ser acrescido está relacionado ao ajuste das séries, a partir dos valores apresentados, as estações de Aracaju e Itabaianinha são ajustadas pela distribuição Fréchet ( $\square \square \square$ ), enquanto a estão de Propriá pode ser ajustada por uma distribuição Weibull negativa $(\square \square \square \square$.

Tabela 2 - Resultados dos parâmetros da distribuição GEV e precipitação máxima diária anual.

\begin{tabular}{ccccc}
\hline Estação & $\square \square(\mathrm{mm})$ & $\square \square(\mathrm{mm})$ & $\square$ & Chuva máxima diária (mm) \\
\hline Aracaju & $60,95 \pm 5,70$ & $22,83 \pm 4,60$ & 0,19 & 157 (abril de 2012) \\
Itabaianinha & $49,52 \pm 4,47$ & $18,01 \pm 3,48$ & 0,11 & 119 (maio de 1997) \\
Propriá & $51,01 \pm 4,84$ & $19,70 \pm 3,61$ & $-0,07$ & 113 (março de 2008) \\
\hline
\end{tabular}

A partir daí, foram obtidos diagnósticos da distribuição GEV via gráficos de probabilidade, quantis-quantis, nível de retorno e de densidade com o propósito de avaliar cada série utilizada. Assim, com base nos resultados obtidos, nenhuma das três estações de estudo conseguiram ser modeladas por uma distribuição normal, ou seja, não passaram no teste de normalidade, como prova da não-normalidade é a presença de outliers nos gráficos de quantis-quantis (lado superior direito das Figuras 8, 9 e 10), impossibilitando que a série se mantenha próximo a reta.

Analisando a estação de Aracaju, constata-se pelo gráfico de densidade (Figura 8, gráfico inferior direito) que a distribuição dos extremos diários anuais de precipitação estão concentradas entre os valores de $40 \mathrm{~mm}$ e $80 \mathrm{~mm}$, além disso, um aumento gradativo entre 120-160 mm, demonstrando que o ajuste da série é realizado pela distribuição Fréchet, provocado pela concentração de observações nas extremidades das distribuições (sendo este do lado direito ou esquerdo), também chamadas de caudas pesadas.

A partir do gráfico de nível de retorno ou de recorrência (Figura 8, gráfico inferior esquerdo), verifica-se que poderá ocorrer pelo menos um evento com intensidades entre 150$170 \mathrm{~mm}$ nos próximos 5-10 anos. Já o diagnóstico da distribuição GEV para estação de Itabaianinha (Figura 9) apresenta algumas semelhanças referente a não se adaptar a uma distribuição normal e a presença de outliers, que foram anteriormente discutidas (Figura 9, gráficos superiores direito e esquerdo, respectivamente). 


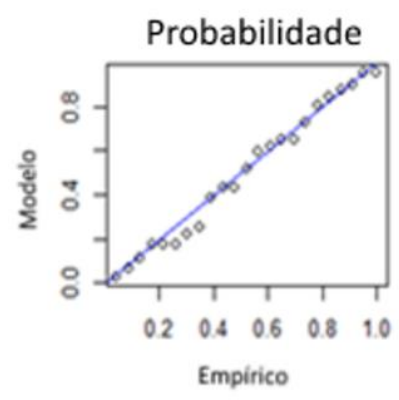

Nível de Retorno

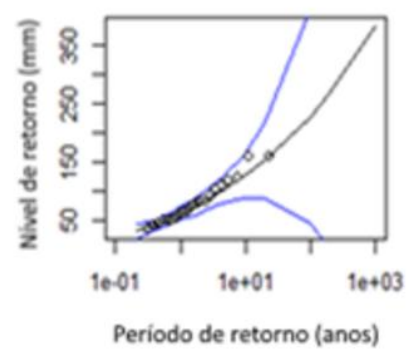

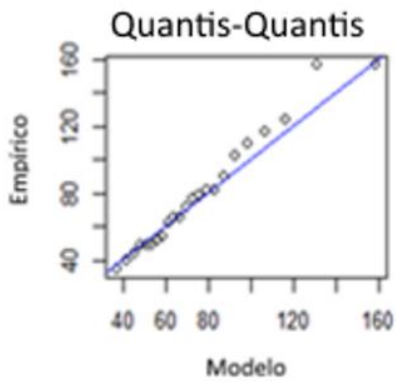

Densidade

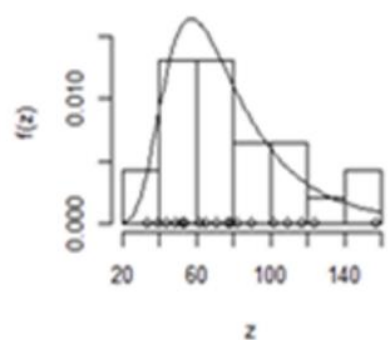

Figura 8 - Diagnóstico dos extremos diários anuais de precipitação via distribuição GEV para o período chuvoso (março a junho) do período de 1993-2015, para a estação de Aracaju.

A frequência dos extremos de precipitação da estação de Itabaianinha está localizada entre as faixas 40mm e $60 \mathrm{~mm}$. Quanto aos níveis e períodos de retorno (Figura 9, gráfico inferior direito), constata-se que ocorrerá pelo menos um evento com intensidades entre 100$120 \mathrm{~mm}$ nos próximos 5-10 anos.
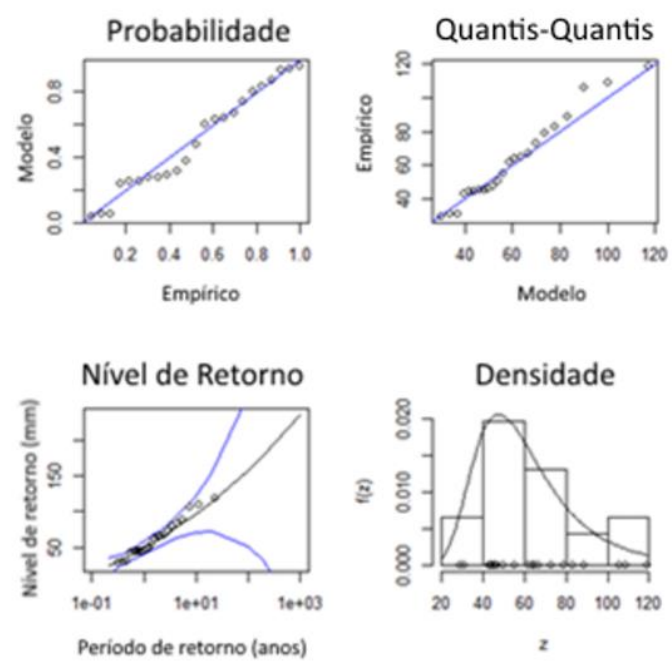

Figura 9 - Diagnóstico dos extremos diários anuais de precipitação via distribuição GEV para o período chuvoso (março a junho) do período de 1993-2015, para a estação de Itabaianinha. 
O diagnóstico obtido a partir dos dados da estação de Propriá (Figura 10) mostra que a distribuição de frequência dos extremos diários anuais de precipitação está entre a faixa de 60-80 mm, constatado pelo gráfico de densidade (Figura 10, gráfico inferior direito).
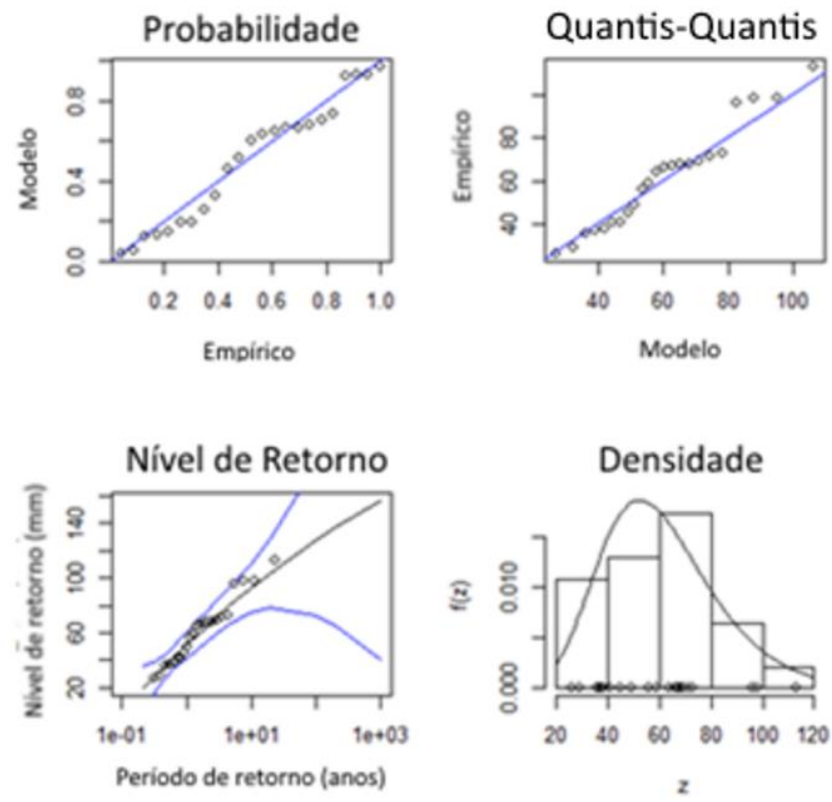

Figura 10 - Diagnóstico dos extremos diários anuais de precipitação via distribuição GEV para o período chuvoso (março a junho) do período de 1993-2015, para a estação de Propriá.

Verifica-se que a cauda do lado direito da série demonstra um decaimento abrupto, muito característico da distribuição Weibull negativa. O gráfico de nível de retorno (Figura 10, gráfico inferior esquerdo) indica que pode ocorrer pelo menos um evento com intensidades entre 100-120 mm nos próximos 5-10 anos.

\section{CONCLUSÕES}

Com base nos resultados apresentados, conclui-se que a variabilidade anual de precipitação para o estado de Sergipe verificada pelo Índice de Anomalia de Chuva (IAC) apresentou oscilações entre anos de anomalias positivas e negativas. Em anos subsequentes da ocorrência de El Niños houve uma diminuição nos totais de precipitação e também nos seus 
extremos verificado pelas três estações. No ano de 2012, ano posterior ao La Niña de intensidade moderada (ocorrido em 2011), ocorreu na maior redução no índice total anual de precipitação, motivado pelas fortes anomalias positivas de TSM na região Tropical do Atlântico.

Já a análise dos extremos diários de precipitação pela distribuição GEV, constatou que os parâmetros de localização e de escala para as três estações meteorológicas possuíam comportamentos similares, com pequenas variações, em torno de 49,52 $\mathrm{mm}$ e $60,95 \mathrm{~mm}$, e entre $19,70 \mathrm{~mm}$ e $22,83 \mathrm{~mm}$, respectivamente. Já o parâmetro de forma, as estações de Aracaju e Itabaianinha se ajustam a uma distribuição Fréchet, enquanto a estação de Propriá se ajusta a uma distribuição Weibull negativa. Os períodos de retorno mostraram que pode ocorrer pelo menos uma chuva acima de $150 \mathrm{~mm}$ para a estação de Aracaju, e valores entre 100-120 mm para as estações de Itabaianinha e Propriá nos próximos 5-10 anos.

\section{AGRADECIMENTOS}

Os autores agradecem ao INMET pela concessão dos dados para a realização deste estudo. O segundo e terceiro autores agradecem as bolsas de PNPD da CAPES e do CNPq, respectivamente. Aos revisores anônimos que contribuíram com recomendações e sugestões para a melhoramento deste trabalho.

\section{REFERÊNCIAS BIBLIOGRÁFICAS}

ANDREOLI, R.V.; KAYANO, M. T. Tropical Pacific and South Atlantic effects on precipitation variability over Northeastern Brazil. Int. J. Climatol., DOI. 10.1002/JOC.1341, v. 26, n.13, p. 1895-1912, 2006.

BEIRLANT, J.; GOEGEBEUR, Y.; SEGERS, J.; TEUGELS, J. Statistics of extremes: theory and applications, 497p., John Wiley \& Sons, 2006.

BRAGA, T. M.; OLIVEIRA, E. L. de; GIVISIEZ, G. H. N. Avaliação de metodologias de mensuração de risco e vulnerabilidade social a desastres naturais associados à mudança climática. São Paulo em Perspectiva, v. 20, n. 1, p. 81-95, 2006. 
CAVALCANTI, I.F.A. Large scale and synoptic features associated with extreme precipitation over South America: A review and case studies for the first decade of the 21st century. Atmospheric Res., v. 118, p. 27-40, 2012.

COLES, S.; BAWA, J.; TRENNER, L.; DORAZIO, P. An introduction to statistical modeling of extreme values (Vol. 208), 209 p., London: Springer, 2001.

CORREIA FILHO, W. L. F. Modelos de dispersão para extremos de precipitação. Estudo de caso: o nordeste do Brasil. 2014. 125f. Tese (Doutorado em Ciências Climáticas) Centro de Ciências Exatas e da Terra, Universidade Federal do Rio Grande do Norte, Natal, 2014.

DE SOUSA GURJÃO, C. D.; CORREIA, M. de F.; CHAVES FILHO, J. B.; SILVA ARAGÃO, M. R. Influência do Enos (El Niño-Oscilação Sul) no Regime Hidrológico do Rio São Francisco: uma Análise em Regiões com Fortes Pressões Antrópicas Rev. Bras. Geogr. Fís., v. 5, n. 4, p. 774-790, 2012.

DOS SANTOS, E. P. dos; CORREIA, M. F.; ARAGAO, M.R.S.; ARAUJO, L. E.; SILVA, F.D.S. Padrão Mensal de Anomalias de Precipitação: Uma Análise Estatística de Eventos Hidrológicos Extremos nas Sub-Bacias do Rio São Francisco. Rev. Bras. Geogr. Fís., v. 6, n. 5, p. 1193-1207, 2013.

DINIZ, M. T. M.; MEDEIROS, S.C.; CUNHA, C. J. Sistemas Atmosféricos Atuantes e Diversidade Pluviométrica em Sergipe. Instituto de Estudos Sócio-Ambientais Bol. Goiano de Geogr., v. 34, n. 1. 17 - 34, 2014.

EASTERLING, D. R.; EVANS, J. L.; GROISMAN, P. Y.; KARL, T. R. Observed Variability and Trends in Extreme Climate Events: A Brief Review. Bull. of the Amer. Meteorol. Soc., v. 81, n. 3, p. 417-425, 2000.

FENG, S.; NADARAJAH, S.; HU, Q. Modeling annual extreme precipitation in China using the generalized extreme value distribution. J. Meteorol. Soc. Japan, v.85, n. 5, p.599-613, 2007.

FERNANDES, D. S.; HEINEMANN, A. B.; PAZ, R. L. DA.; AMORIM, A. DE O.; CARDOSO, A. S. Índices para a quantificação da seca. Santo Antônio de Goiás: 
Embrapa Arroz e Feijão, 2009. FOLLAND, C. K. et al., Observed climate variability and change. Climate change: The scientific basis. Contribution of Working Group I to the Third Assessment Report of the Intergovernmental Panel on Climate Change, Eds. J. T. Houghton, Y. Ding, D. J. Griggs, M. Noguer, P. van der Linden, X. Dai, K. Maskell, and C. I. Johnson. Cambridge University Press, p. 525-582, 2001.

FREITAS, M. A. S. Um sistema de suporte à decisão para o monitoramento de secas meteorológicas em regiões semi-áridas. Rev. Tecnologia, v.19, p.84-95, 2005.

GURJEL, J. J. S. Sobre a produção de pescado dos açudes públicos do Semiárido do Nordeste Brasileiro. 1986. Organizacion de las Naciones Unidas para la Agricultura y la Alimentacion - FAO. Disponível: http://www.fao.org/docrep/008/ad772s/AD772S05.htm. Acessado: 16 dez. 2015.

HASTENRATH, S. Exploring the climate problems of Brazil's Nordeste: a review. Climatic Change, v.112, p.243-251, 2011, doi:10.1007/s10584-011-0227-1.

IDAliNO, R. C. L.; OliVEIRA, P. S.; LUCIO, P. S. Modelagem de Extremos Meteorológicos via GEV e GPD - Uma análise comparativa de algumas capitais brasileiras. Rev. Bras. Estat., v.70, p.33-56, 2009.

JENKINSON, A. F. The frequency distribution of the annual maximum (or minimum) values of meteorological elements. Q. J. Roy. Meteor. Soc., v. 81, n. 348, p. 158-171, 1955.

KEYANTASH, J.; DRACUP, J. A. The quantification of drought: An analysis of drought indices, Bull. Am. Meteorol. Soc., 83(8), 1167 - 1180, 2002.

KUCHARSKI, F.; POLZIN, D.; HASTENRATH, S. Teleconnection Mechanisms of Northeast Brazil Droughts: Modeling and Empirical Evidence. Rev. Bras. Meteor., v.23, n. 2, p.115-125, 2008.

LIEBMANN, B.; KILARDIS, G. N.; ALluRED, D.; VERA, C. S.; JONES, C.; CARVAlHO, L. M. V.; BLADÉ, I.; GONZÁleS, P. L. M. Mechanisms Associated with Large Daily Precipitation Events in Northeast Brazil, J. Climate, v.24, p.1589-1609, 2011.

MARENGO, J. A. Água e mudanças climáticas. Estudos Avançados, v. 22, n. 63, 2008. 
MEEHL, G.A.; TEBALDI, C. More intense, more frequent, and longer lasting heat waves in the 21st century. Science, v. 305, p. 994-997, 2004.

MOLION, L. C. B.; BERNARDO, S. O. Uma revisão da dinâmica das chuvas no nordeste brasileiro. Rev. Bras. Meteor., v. 17, n. 1, p. 1-10, 2002.

RAO, V. B.; HADA, K.; HERDIES, D. L. On the severe drought of 1993 in north-east Brazil. Int. J. Climatol., 15: 697-704, 1995. doi: 10.1002/joc.3370150608.

PELlEGRINO, G. Q.; ASSAD, E. D.; MARIN, F. R.. Mudanças climáticas globais e a agricultura no Brasil. Rev. Multiciência, Campinas, n. 8, p. 139-162, 2007.

PEREIRA, E. R. R.; FREITAS, F. C.; ANDRADE, A. R. S.; SOUSA, V. G. de. Variabilidade do número de dias chuvosos no estado de Sergipe-SE. Pesquisa Aplicada \& Agrotecnologia, v.4, n.1, p.45-65, 2011.

RODRIGUES, R. R.; MCPHADEN, M. J. Why did the 2011-2012 La Niña cause a severe drought in the Brazilian Northeast?. Geophys. Res. Lett., v. 41, n. 3, p. 1012-1018, 2014.

ROOY, M. P.; VAN, A. Rainfall Anomaly Index Independent of Time and Space, Notos, 14, 43, 1965.

SANTOS, E. C. A. DOS; ARAUJO, L. E. DE; MARCELINO, A. D. S.. Análise climática da Bacia Hidrográfica do Rio Mamanguape. Rev. Bras. Eng. Agríc. Amb., Campina Grande, v. 19, n. 1, p. 9-14, 2015.

SCARROTT, C.; MACDONALD, A. A review of Extreme Value Threshold Estimation and uncertainty quantification. REVSTAT, v.10, p.33-60, 2012.

SILVA, V. P. R.; PEREIRA, E. R. R., ALMEIDA, R. S. R. Estudo da variabilidade anual e interanual da precipitação na região Nordeste do Brasil. Rev. Bras. Meteor., São Paulo, v. 27, n. 2, p. 163-172, 2012.

SKANSI, M. M.; BRUNET, M.; SIGRÓ, J.; AGUILAR, E.; GROENING, J. A. A.; BENTANCUR, O. J.; ROJAS, C. O. Warming and wetting signals emerging from analysis of changes in climate extreme indices over South America. Global Planet Change, v. 100, p. 295-307, 2013. 
TOMINAGA, L. K; SANTORO, J; AMARAL, R. (ORG.). Desastres Naturais Conhecer para Prevenir, 196 p., $1^{\circ}$ Ed. Instituto Geológico. São Paulo, 2009.

TRENBERTH, K. E. Changes in precipitation with climate change. Climate Res., v. 47, n. 1, p. $123,2011$.

TRENBERTH, K. E. 2012. Framing the way to relate climate extremes to climate change. Climatic Change, v.115, p. 283-290, 2012.

TUCCI, C. E. M. Mudanças climáticas e impactos sobre os recursos hídricos no Brasil. Ciência \& Ambiente, Santa Maria, RS, n. 34, p. 137-156, 2007.

WMO - WORLD METEOROLOGICAL ORGANIZATION. Guidelines on Analysis of extremes in a changing climate in support of informed decisions for adaptation, $52 \mathrm{p}$., 2009. 